九州大学学術情報リポジトリ

Kyushu University Institutional Repository

\title{
THE ERDÖS-TURÁN LAW FOR MIXTURES OF DIRICHLET PROCESSES (II)
}

Yamato, Hajime

Kagoshima University : Professor Emeritus

https://doi.org/10.5109/1798146

出版情報: Bulletin of informatics and cybernetics. 46, pp.47-51，2014-12. Research Association of Statistical Sciences

バージョン:

権利関係 : 
THE ERDÖS-TURÁN LAW FOR MIXTURES OF DIRICHLET

PROCESSES (II)

by

Hajime Yamato

Reprinted from the Bulletin of Informatics and Cybernetics

Research Association of Statistical Sciences, Vol. 46

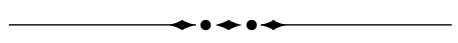

FUKUOKA, JAPAN

2014 


\title{
THE ERDŐS-TURÁN LAW FOR MIXTURES OF DIRICHLET PROCESSES (II)
}

By

\author{
Hajime Yамато*
}

\begin{abstract}
Let a random distribution $\mathcal{P}$ on the real line $\mathbb{R}$ have the mixture of Dirichlet processes. Let $S^{(n)}=\left(S_{1}, \cdots, S_{n}\right)$ be the random partition of the positive integer $n$ based on a sample of size $n$ from $\mathcal{P}$. For the order $O_{n}\left(S^{(n)}\right)$ of $S^{(n)}$, Yamato (2013) gives the asymptotic distribution of the statistic $\log O_{n}\left(S^{(n)}\right) / \log ^{2} n$ and the rate $O\left(1 / \log ^{1 / 3} n\right)$ of its convergence. In this pager we give the Edgeworth expansions for the statistic with the rates $O\left(1 / \log ^{2 / 5} n\right)$ and $O\left(1 / \log ^{3 / 7} n\right)$. In addition, we correct the errors of the proofs of the lemmas 2.5 and 2.6 of Yamato (2013).
\end{abstract}

Key Words and Phrases: Edgeworth expansion, Erdős-Turán law, Fourier transform, mixture of Dirichlet processes, order of partition, random partition, smoothing lemma.

\section{Introduction}

Let $G_{0}$ be a continuous distribution on the real line $\mathbb{R}$ and $\mathcal{B}$ be the $\sigma$-field which consists of the subsets of $\mathbb{R}$. Let $\theta$ be a positive random variable having a distribution $\gamma$. We suppose that a random distribution $\mathcal{P}$ have the mixture of Dirichlet process $\mathcal{D}\left(\theta G_{0}\right)$ on $(\mathbb{R}, \mathcal{B})$ with the mixing distribution $\gamma$ (for the mixture of Dirichlet process, see Antoniak (1974)). For a sample of size $n$ from the random distribution $\mathcal{P}, S_{1}$ denotes the number of observations which occur only once, $S_{2}$ the number of observations which occur exactly twice, $\ldots$ and so on. For the random partition $S^{(n)}=\left(S_{1}, \cdots, S_{n}\right)$ of the positive integer $n$, the order $O_{n}\left(S^{(n)}\right)$ denotes l.c.m. $\left\{j: S_{j}>0(j=1,2, \cdots, n)\right\}$, where l.c.m. represents the least common multiple. Let $H$ be the distribution functions (d.f.) of $\theta / 2$. For the convergence of the statistic $O_{n}\left(S^{(n)}\right) / \log ^{2} n$, Yamato (2013) gives

$$
\sup _{-\infty<x<\infty}\left|P\left(\frac{\log O_{n}\left(S^{(n)}\right)}{\log ^{2} n} \leq x\right)-H(x)\right|=O\left(\frac{1}{\log ^{1 / 3} n}\right) .
$$

In the section 2 we give the Edgeworth expansion for the statistic $O_{n}\left(S^{(n)}\right) / \log ^{2} n$ with the rate $O\left(1 / \log ^{2 / 5} n\right)$, which is the proposition 2.1. In the section 3 , we give the Edgeworth expansion with the rate $O\left(1 / \log ^{3 / 7} n\right)$, which is the proposition 3.1. In the section 4, for the lemmas 2.5 and 2.6 of Yamato (2013), the errors of their proofs are corrected.

\footnotetext{
* Emeritus of Kagoshima University, Take 3-32-1-708 Kagoshima 890-0045 Japan.
} 


\section{The Edgeworth expansion with the rate $O\left(1 / \log ^{2 / 5} n\right)$}

Suppose that $E_{\gamma}\left(\theta^{2}\right)$ exist, where $E_{\gamma}$ denotes the expectation with respect to the distribution $\gamma$. Let $h$ be the bounded density function of the d.f. $H$ (or of the random variable $\theta / 2$ ). For the smoothing lemma (see, for example, Petrov (1995; Theorem 5.2)) used in the proof of the proposition 2.1, we suppose the followings: (i) $h$ be twice differentiable, and $\{x h(x)\}^{\prime}\left(=h(x)+x h^{\prime}(x)\right)$ be of bounded variation, (ii) $h^{\prime}(x)$ and $x h^{\prime \prime}(x)$ be bounded, that is, $\{x h(x)\}^{(2)}$ be bounded and (iii) $h(x)=0, x h^{\prime}(x)=0$ for $x=0$ and $x h^{\prime}(x) \rightarrow 0$ as $x \rightarrow+\infty$. We also use the following lemma.

Lemma 2.1. (Petrov (1995; Lemma 1.8)) Let $X$ and $W$ be arbitrary random variables, and let $F(x)$ be the distribution functions of $X$. If $T(x)$ is an arbitrary function defined on the real line, then for every real $x$ and every positive $\varepsilon$

$$
|P(X+W \leq x)-T(x)| \leq K+L+P(|W|>\varepsilon),
$$

where $K=\max \{|F(x+\varepsilon)-T(x+\varepsilon)|,|F(x-\varepsilon)-T(x-\varepsilon)|\}$ and $L=\max \{\mid$ $T(x+\varepsilon)-T(x)|| T,(x-\varepsilon)-T(x) \mid\}$.

We use the same notations as Yamato (2013), except for $H$ and $h$. Then, we have

Proposition 2.2.

$$
\sup _{-\infty<x<\infty}\left|P\left(\frac{\log O_{n}\left(S^{(n)}\right)}{\log ^{2} n} \leq x\right)-\left[H(x)+\frac{1}{3 \log n}\left\{h(x)+x h^{\prime}(x)\right\}\right]\right|=O\left(\frac{1}{\log ^{2 / 5} n}\right) .
$$

Proof. At first, we note the following relations.

$$
\frac{1}{\log ^{2} n} \sum_{j=1}^{n} \frac{\log j}{j}=\frac{1}{2}+\frac{c_{1}}{\log ^{2} n}, \frac{1}{\log ^{i+1} n} \sum_{j=1}^{n} \frac{\log ^{i} j}{j}=\frac{1}{i+1}+\frac{c_{i}}{\log ^{i+1} n} \quad(i=2,3),
$$

where $c_{i}(i=1,2,3)$ denote generic positive constants. We use also $c$ as a generic constant. By these relations we get

$$
\sum_{j=1}^{n} \frac{1}{j}\left(e^{i t \frac{\log j}{\log ^{2} n}}-1\right)=i \frac{1}{2} t-\frac{t^{2}}{6 \log n}+c \frac{|t|}{\log ^{6 / 5} n} \quad\left(|t| \leq \log ^{2 / 5} n\right) .
$$

Given $\theta$, let $Z_{1}, \cdots, Z_{n}$ be independent and $Z_{j}$ have the Poisson distribution with mean $\theta / j(j=1, \cdots, n)$. For $Z^{(n)}=\left(Z_{1}, \cdots, Z_{n}\right)$, we put

$$
O_{n}\left(Z^{(n)}\right)=\text { l.c.m. }\left\{j: Z_{j}>0(j=1,2, \cdots, n)\right\}, \quad T_{n}\left(Z^{(n)}\right)=\prod_{j=1}^{n} j^{Z_{j}}
$$

and $\mu_{n}(\theta)=E\left[\log T_{n}\left(Z^{(n)}\right)-\log O_{n}\left(Z^{(n)}\right) \mid \theta\right]$. We put

$$
S_{1 n}^{*}=\frac{\log T_{n}\left(Z^{(n)}\right)}{\log ^{2} n}, \quad S_{2 n}^{*}=\frac{\log O_{n}\left(Z^{(n)}\right)+\mu_{n}(\theta)}{\log ^{2} n} \quad \text { and } \quad S_{3 n}^{*}=\frac{\log O_{n}\left(S^{(n)}\right)}{\log ^{2} n} .
$$
have

Then, for the characteristic function (c.f.) $f_{n}$ of $S_{1 n}^{*}=\sum_{j=1}^{n} Z_{j} \log j / \log ^{2} n$, we

$$
\begin{aligned}
f_{n}(t) & =E_{\gamma} \exp \left\{\theta \sum_{j=1}^{n} \frac{1}{j}\left(e^{i t \frac{\log j}{\log ^{2} n}}-1\right)\right\}=E_{\gamma}\left[e^{i \frac{\theta}{2} t} \exp \left\{-\theta \frac{t^{2}}{6 \log n}+c \theta \frac{|t|}{\log ^{6 / 5} n}\right\}\right] \\
& =E_{\gamma} e^{i \frac{\theta}{2} t}-\frac{t^{2}}{3 \log n} E_{\gamma}\left(\frac{\theta}{2} e^{i \frac{\theta}{2} t}\right)+c_{1} \frac{|t|}{\log ^{4 / 5} n}, \quad\left(|t| \leq \log ^{2 / 5} n\right) .
\end{aligned}
$$


Let the c.f. of $\theta / 2$ be $\varphi_{H}(t)=\int_{-\infty}^{\infty} e^{i x t} h(x) d x=\int_{-\infty}^{\infty} e^{i x t} d H(x)$. Since $\varphi_{H}(t)$ be the Fourier transform of $h,-t^{2} \int_{-\infty}^{\infty} e^{i x t} x h(x) d x$ is the Fourier transform of $\{x h(x)\}^{\prime \prime}$. Or, $\varphi_{H}(t)=E_{\gamma} e^{i \frac{\theta}{2} t}$ corresponds to $H$ and $-t^{2} \int_{\infty}^{\infty} e^{i x t} x h(x) d x=-t^{2} E_{\gamma}\left(\frac{\theta}{2} e^{i \frac{\theta}{2} t}\right)$ to $\{x h(x)\}^{\prime}$. Therefore, by the smoothing lemma, we have

$$
\begin{aligned}
\sup _{x} \mid P\left(S_{1 n}^{*} \leq x\right)-[H(x)+ & \left.\frac{1}{3 \log n}\left\{h(x)+x h^{\prime}(x)\right\}\right] \mid \\
& \leq \frac{c_{1}}{\log ^{4 / 5} n} \int_{0}^{\log ^{2 / 5} n} d t+\frac{c_{2}}{\log ^{2 / 5} n}=O\left(\frac{1}{\log ^{2 / 5} n}\right) .
\end{aligned}
$$

This corresponds to Proposition 2.3 of Yamato (2013). Next, we derive the results with $S_{2 n}^{*}$ and $S_{3 n}^{*}$ similar to (3), instead of $S_{1 n}^{*}$.

(I) We use Lemma 2.1 by taking $X=S_{1 n}^{*}, W=S_{2 n}^{*}-S_{1 n}^{*}, T(x)=H(x)+\{h(x)+$ $\left.x h^{\prime}(x)\right\} /(3 \log n)$, and $K \leq \sup _{-\infty<x<\infty}\left|P\left(S_{1 n}^{*} \leq x\right)-T(x)\right|=O\left(1 / \log ^{2 / 5} n\right)$. By (15) of the section 4, we have $P(|W|>\varepsilon)=O\left(1 / \log ^{2} n\right)$ for any $\varepsilon>0$. Since $h^{\prime}$ and $x h^{\prime \prime}$ are bounded, we get $L=O\left(1 / \log ^{2 / 5} n\right)$ with $\varepsilon=1 / \log ^{2 / 5} n$. Thus we have

$$
\sup _{-\infty<x<\infty}\left|P\left(S_{2 n}^{*} \leq x\right)-\left[H(x)+\frac{1}{3 \log n}\left\{h(x)+x h^{\prime}(x)\right\}\right]\right|=O\left(\frac{1}{\log ^{2 / 5} n}\right) .
$$

(II) Again, we use Lemma 2.1 by taking $X=S_{2 n}^{*}$ and $W=S_{3 n}^{*}-S_{2 n}^{*}, K \leq \sup _{-\infty<x<\infty} \mid$ $P\left(S_{2 n}^{*} \leq x\right)-T(x) \mid=O\left(1 / \log ^{2 / 5} n\right)$. $T(x)$ equals to the one of the above paragraph. With $\varepsilon=1 / \log ^{1 / 2} n$, we have $P(|W|>\varepsilon) \leq O\left(\log \log n / \log ^{1 / 2} n\right)=o\left(1 / \log ^{2 / 5} n\right)$ by (18) of the section 4 and $L=O\left(1 / \log ^{1 / 2} n\right)=o\left(1 / \log ^{2 / 5} n\right)$ by the boundedness of $h^{\prime}$ and $x h^{\prime \prime}$. Thus we have the following (5), which yields (1).

$$
\sup _{-\infty<x<\infty}\left|P\left(S_{3 n}^{*} \leq x\right)-\left[H(x)+\frac{1}{3 \log n}\left\{h(x)+x h^{\prime}(x)\right\}\right]\right|=O\left(\frac{1}{\log ^{2 / 5} n}\right) .
$$

The assumptions about $\theta$ or $\theta / 2(h)$ of the section 2 are satisfied, for example, by the gamma distribution whose density is $h(x)=x^{d-1} e^{-x} / \Gamma(d)(x>0, d>2)$.

\section{The Edgeworth expansion with the rate $O\left(1 / \log ^{3 / 7} n\right)$}

We suppose that $E_{\gamma}\left(\theta^{3}\right)$ exist. In addition to the assumption of the first paragraph of the section 2.1, we suppose that $h$ is differentiable four times. Suppose that $h^{\prime}(x), x h^{(2)}(x)$ and $x^{2} h^{(3)}(x)$ are of bounded variation, and that $h^{(2)}(x), x h^{(3)}(x)$ and $x^{2} h^{(4)}(x)$ are bounded. Suppose that $x h^{(2)}(x)=0, x^{2} h^{(3)}(x)=0$ for $x=0$ and $x h^{(2)}(x)$, $x^{2} h^{(3)}(x) \rightarrow 0$ as $x \rightarrow+\infty$. At first, we note the following relation.

$$
\sum_{j=1}^{n} \frac{1}{j}\left(e^{i t \frac{\log j}{\log ^{2} n}}-1\right)=i \frac{1}{2} t-\frac{t^{2}}{6 \log n}-i \frac{t^{3}}{24 \log ^{2} n}+c \frac{|t|}{\log ^{12 / 7} n} \quad\left(|t| \leq \log ^{3 / 7} n\right) .
$$

Thus, for the c.f. $f_{n}$ of $S_{1 n}^{*}$, we have

$$
\begin{aligned}
f_{n}(t)= & E_{\gamma} e^{i \frac{\theta}{2} t}-\frac{t^{2}}{3 \log n} E_{\gamma}\left(\frac{\theta}{2} e^{i \frac{\theta}{2} t}\right)-i \frac{t^{3}}{12 \log ^{2} n} E_{\gamma}\left(\frac{\theta}{2} e^{i \frac{\theta}{2} t}\right) \\
& +\frac{t^{4}}{18 \log ^{2} n} E_{\gamma}\left[\left(\frac{\theta}{2}\right)^{2} e^{i \frac{\theta}{2} t}\right]+c_{1} \frac{|t|}{\log ^{6 / 7} n}, \quad\left(|t| \leq \log ^{3 / 7} n\right) .
\end{aligned}
$$


Under the Fourier transform, $-i t^{3} E_{\gamma}\left(\frac{\theta}{2} e^{i \frac{\theta}{2} t}\right)$ correponds to $\{x h(x)\}^{(2)}$ and $t^{4} E_{\gamma}\left[\left(\frac{\theta}{2}\right)^{2} e^{i \frac{\theta}{2} t}\right]$ to $\left\{x^{2} h(x)\right\}^{(3)}$. Therefore, by the smoothing lemma, we have

$$
\begin{aligned}
\sup _{x} \mid P\left(S_{1 n}^{*} \leq x\right) & -\left[H(x)+\frac{1}{3 \log n}\left\{h(x)+x h^{\prime}(x)\right\}\right. \\
& \left.+\frac{1}{36 \log ^{2} n}\left\{6 h^{\prime}(x)+9 x h^{(2)}(x)+2 x^{2} h^{(3)}(x)\right\}\right] \mid=O\left(\frac{1}{\log ^{3 / 7} n}\right) .
\end{aligned}
$$

Corresponding to (I) of the section 2, we get $L=O\left(1 / \log ^{3 / 7} n\right)$ with $\varepsilon=1 / \log ^{3 / 7} n$ and the result (7) with $S_{2 n}^{*}$ instead of $S_{1 n}^{*}$. Corresponding to (II) of the section 2, with $\varepsilon=1 / \log ^{1 / 2} n$ we get $P(|W|>\varepsilon) \leq O\left(\log \log n / \log ^{1 / 2} n\right)=o\left(1 / \log ^{3 / 7} n\right)$, $L=O\left(1 / \log ^{1 / 2} n\right)=o\left(1 / \log ^{3 / 7} n\right)$ and the result (7) with $S_{3 n}^{*}$ instead of $S_{1 n}^{*}$. Thus, we have the following.

Proposition 3.1.

$$
\begin{aligned}
\sup _{x} \mid P\left(\frac{\log O_{n}\left(S^{(n)}\right)}{\log ^{2} n} \leq x\right)-\left[H(x)+\frac{1}{3 \log n}\left\{h(x)+x h^{\prime}(x)\right\}\right. \\
\left.+\frac{1}{36 \log ^{2} n}\left\{6 h^{\prime}(x)+9 x h^{(2)}(x)+2 x^{2} h^{(3)}(x)\right\}\right] \mid=O\left(\frac{1}{\log ^{3 / 7} n}\right) .
\end{aligned}
$$

The assumptions about $\theta$ or $\theta / 2(h)$ of the section 3 are satisfied, for example, by the gamma distribution whose density is $h(x)=x^{d-1} e^{-x} / \Gamma(d)(x>0, d>3)$.

\section{Corrections to Yamato (2013)}

In the following, we correct the proofs of Lemma 2.5 and 2.6 of Yamato (2013), which is from the line 6 from the top of page 65 to the line 3 from the bottom of the same page. The numbers of the equations are equal to the ones of Yamato (2013).

By the proposition 2.3 and its proof of Barbour and Tavaré (1994), it holds that

$$
\left.P\left(\mid \log T_{n}\left(Z^{(n)}\right)-\log O_{n}\left(Z^{(n)}\right)-\mu_{n}(\theta)\right)\left|>\varepsilon \log ^{2} n\right| \theta\right)=\theta c_{1 n}+\theta^{2} c_{2 n} \text { for } \forall \varepsilon>0
$$

where $\left.c_{1 n}=O\left((\log \log n)^{2}\right) / \log ^{3} n\right)$ and $c_{2 n}=O\left(1 / \log ^{2} n\right)$. Therefore, under the condition $E_{\gamma} \theta^{2}<\infty$, by (13) and (14) we have

$$
P\left(\left|S_{1 n}^{*}-S_{2 n}^{*}\right|>\varepsilon\right)=O\left(\frac{1}{\log ^{2} n}\right) \quad \text { for } \forall \epsilon>0 .
$$

We use the relation (4) by taking $U=S_{1 n}^{*}, X=S_{2 n}^{*}-S_{1 n}^{*}, H=\gamma^{*}, \eta=O\left(1 / \log ^{1 / 3} n\right)$, and $\varepsilon=O\left(1 / \log ^{1 / 3} n\right)$. By the relation (3) and (15), we obtain

$$
\sup _{-\infty<x<\infty}\left|P\left(S_{2 n}^{*} \leq x\right)-\gamma^{*}(x)\right|=O\left(\frac{1}{\log ^{1 / 3} n}\right) .
$$

Proof of Lemma 2.6 By the relation (2.1) and (2.2) of Barbour and Tavaré (1994), we have

$$
\left|S_{2 n}^{*}-S_{3 n}^{*}\right| \leq\left|\frac{\log O_{n}\left(Z^{(n)}\right)-\log O_{n}\left(S^{(n)}\right)}{\log ^{2} n}\right|+\frac{\left|\mu_{n}(\theta)\right|}{\log ^{2} n} \leq Y+\frac{\mu_{n}(\theta)}{\log ^{2} n}, \text { given } \theta
$$


where $Y=\left(Y_{n}+1\right) / \log n, E\left(Y_{n}\right)=E_{\gamma}\left(E\left(Y_{n} \mid \theta\right)\right) \leq E_{\gamma} \theta^{2}$ and $E_{\gamma} \mu_{n}(\theta)=O(\log n \log \log n)$, where $(0 \leq) \mu(\theta)=\theta \log n \log \log n+c \theta^{2} \log n$ (Barbour and Tavaré (1994; p.171)). Thus, by the Markov's inequality and (17) we have

$$
P\left(\left|S_{2 n}^{*}-S_{3 n}^{*}\right|>\varepsilon\right) \leq P\left(|Y|>\frac{\varepsilon}{2}\right)+P\left(\frac{\left|\mu_{n}(\theta)\right|}{\log ^{2} n}>\frac{\varepsilon}{2}\right) \leq c \frac{\log \log n}{\varepsilon \log n} \quad \text { for } \forall \varepsilon>0 .
$$

We use the relation (4) by taking $U=S_{2 n}^{*}, X=S_{3 n}^{*}-S_{2 n}^{*}, H=\gamma^{*}, \eta=O\left(1 / \log ^{1 / 3} n\right)$, and $\varepsilon=O\left(1 / \log ^{1 / 2} n\right)$. By the relation (16) and (18) with $\log \log n /(\varepsilon \log n)=o\left(1 / \log ^{1 / 3} n\right)$, we obtain

$$
\sup _{-\infty<x<\infty}\left|P\left(S_{3 n}^{*} \leq x\right)-\gamma^{*}(x)\right|=O\left(\frac{1}{\log ^{1 / 3} n}\right) .
$$

\section{Acknowledgement}

The author is grateful to the referee for his careful reading and useful comments.

\section{References}

Antoniak, C. E. (1974). Mixtures of Dirichlet processes with applications to Bayesian nonparametric problems. Ann. Statist., 2, 1152-1174.

Barbour, A.D. and Tavaré, S. (1994). A rate for the Erdős-Turán law. Combin. Probab. Comput., 3, 167-176.

Petrov, V. (1995). Limit theorems of probability theory. New York: Oxford Univ. Press.

Yamato, H. (2013). The Erdős-Turán law for mixtures of Dirichlet processes. Bull. Inform. Cyber., 45, 59-66.

Received May 20, 2014

Revised October 20, 2014 